\title{
Roteamento e Alocação de Núcleo e Espectro com Ciência de Fragmentação e Crosstalk em SDM-EON
}

\author{
Edson Rodrigues ${ }^{1}$, Gustavo B. Figueiredo ${ }^{2}$, Juliana de Santi ${ }^{3}$, Helder Oliveira ${ }^{1}$ \\ ${ }^{1}$ Instituto de Ciências Exatas e Naturais - Universidade Federal do Pará (UFPA) \\ ${ }^{2}$ Departamento de Ciência da Computação - Universidade Federal da Bahia (UFBA) \\ ${ }^{3}$ Departamento de Informática - Universidade Tecnológica Federal do Paraná (UTFPR) \\ edson.rodrigueseitec.ufpa.br ${ }^{1}$, gustavoddcc.ufba.br ${ }^{2}$, \\ jsanti@utfpr.edu.br ${ }^{3}$, heldermay@ufpa.br ${ }^{1}$
}

\begin{abstract}
The explosive growth of traffic on the backbone of the Internet reflects advancements in technologies that make it possible to popularize highbandwidth, low-latency network applications. However, traffic growth and network capacity are rarely compatible. In this sense, the Spatial Division Multiplexing technology in Elastic Optical Networks has shown to be quite promising due to its capacity expansion and requests adjustment properties. This paper proposes a modulation, spectrum, and core allocation and routing algorithm for SDM-EON, focusing on reducing the request blocking rate via medium crosstalk and fragmentation reduction strategies. The results obtained demonstrate the high efficiency of the proposed algorithm in establishing connections compared to similar algorithms in the literature.
\end{abstract}

Resumo. O crescimento explosivo do tráfego no backbone da Internet reflete o avanço nas tecnologias que possibilitam a popularização de aplicativos de rede de alta largura de banda e baixa latência. Contudo, o crescimento do tráfego e a capacidade da rede raramente se mostram compatíveis. Neste sentido, a tecnologia de Multiplexação por Divisão Espacial em Redes Ópticas Elásticas tem se mostrado bastante promissora devido às suas propriedades de ampliação da capacidade e ajuste de requisições. Este artigo propõe um algoritmo de roteamento e alocação de modulação, espectro e núcleo para as SDM-EON, com enfoque na redução da taxa de bloqueio de requisições via estratégias de redução de crosstalk médio e fragmentação. Os resultados obtidos demonstram a alta eficiência do algoritmo proposto no estabelecimento de conexões em comparação com algoritmos semelhantes na literatura.

\section{Introdução}

O tráfego IP global juntamente com o tráfego de dispositivos sem fio e móveis está aumentando a uma taxa de $60 \%$ ao ano [Cisco 2018]. Esse crescimento explosivo do consumo global de dados tem colocado uma enorme pressão sobre as redes de telecomunicações. Neste cenário, as redes baseadas em fibra óptica são uma parte essencial da infraestrutura de redes, sendo responsáveis por conectar milhões de usuários em todo o mundo à Internet. Uma enorme quantidade de dados é transmitida diariamente através dessas redes [Mendinueta et al. 2020]. À medida que a demanda por largura de banda aumenta, impulsionada principalmente pela rápida expansão de dispositivos conectados e 
pelo crescimento de serviços e aplicativos baseados em nuvem, surge a necessidade de conexões mais rápidas e confiáveis. O grande problema dessa conjuntura é que uma fibra monomodo, fibra amplamente utilizada nas redes ópticas existentes, possui capacidade física limitada e há uma tendência dessa capacidade não ser suficiente em um futuro próximo [Secondini 2020].

As fibras monomodo convencionais serviram como meio de transmissão confiável nos últimos 30 anos, possibilitando que os operadores de rede acompanhassem o aumento do tráfego de dados por meio de uma sequência de inovações técnicas associadas ao uso cada vez maior da capacidade de transmissão da fibra [Oliveira and da Fonseca 2019]. No entanto, é amplamente reconhecido que a capacidade máxima de transmissão de uma fibra monomodo está se aproximando rapidamente do seu limite fundamental de transporte de dados, imposto em parte por uma combinação da não linearidade da fibra e pela largura de banda do amplificador da fibra [Bonani et al. 2019]. Aumentar o número de fibras é uma solução que envolve um alto custo além de requerer requisitos significativos de consumo de energia que podem ser insustentáveis. Recentemente, a tecnologia SDM (Space-Division Multiplexing) tem atraído atenção na comunidade de comunicação por ser uma tecnologia promissora para aumentar significativamente a capacidade de transmissão da fibra e reduzir o custo total por bit transmitido [Winzer 2020].

Uma das estratégias utilizadas para acomodar o crescimento do tráfego de dados é a implementação das EON (Elastic Optical Networks) com o aproveitamento de suas características de granularidade do espectro dividido em slots, que contribui para a redução na quantidade de recursos ociosos na rede. Aliado ao mecanismo de ampliação de capacidade gerada pela tecnologia SDM, as SDM-EON (Space-Division Multiplexing Elastic Optical Networks) se apresentam como uma solução viável para suprir as demandas futuras [Trindade and da Fonseca 2020]. O surgimento dessas redes dá origem a novos desafios para a gestão de recursos devido ao aumento da complexidade provocado pela utilização de fibras multinúcleo. Junto com a SDM-EON surge o problema do RSCA (Routing, Spectrum, and Core Allocation), que refere-se a alocação dos recursos disponíveis na rede, realizando a seleção do núcleo a ser utilizado, mantendo as restrições desse tipo de rede conhecidas como continuidade e contiguidade do espectro, que garante que as transmissões ocorram através de um único núcleo durante todo o percurso e os slots tomados para a requisição devem ser contíguos dentro do núcleo selecionado [Zhu et al. 2021]. Para complementar o problema RSCA, existe a adição de uma característica na transmissão que habilita o maior número de bits por transmissão que é a aplicação de modulação adaptativa [Iyer 2020]. O conjunto do problema RSCA e a modulação adaptativa dá origem ao problema RMSCA (Routing, Modulation, Spectrum, and Core Allocation) para SDM-EON.

Para prover melhor alocação e utilização do espectro em SDM-EON algumas abordagens podem ser levadas em consideração na alocação dos recursos. Dentre essas abordagens destacam-se a verificação da fragmentação do espectro, presente em redes elásticas, e a geração de crosstalk médio presente em redes multinúcleos. A fragmentação é um problema comum decorrente da alocação e remoção dinâmica das conexões [Xiong et al. 2020]. Esse fenômeno caracteriza-se pelo surgimento de pequenos intervalos no espectro que não são suficientes para alocar outras requisições, provocando a subutilização do recurso. Outro desafio das redes que empregam fibras multinúcleo é a in- 
terferência entre núcleos conhecida como crosstalk [Paira et al. 2020]. Essa interferência ocorre quando um mesmo espectro de frequência é utilizado em núcleos adjacentes dentro da fibra, podendo comprometer a decodificação do sinal caso o nível de interferência seja acima do aceitável.

Neste contexto, este artigo introduz um algoritmo RMSCA chamado Roteamento e Alocação de Modulação, Núcleo e Espectro com Ciência de Fragmentação e Crosstalk em SDM-EON (REGARD). O algoritmo proposto busca reduzir a taxa de bloqueio de requisições através da verificação da fragmentação e do crosstalk médio. O REGARD proporciona uma melhor utilização do espectro de frequência da rede considerando diversas características na seleção dos recursos da conexão. No processo de alocação, primeiramente é encontrado um caminho de um ponto a outro e os recursos para acomodar a requisição são buscados. A definição de quais slots de frequência e qual núcleo serão utilizados é feita dinamicamente levando em consideração o estado da rede, sempre buscando alocar mais requisições e controlar os níveis de fragmentação e crosstalk.

O restante desse trabalho está organizado da seguinte forma: na Seção 2 está uma visão geral do estado-da-arte acerca de SDM-EON. A Seção 3 apresenta as topologias de rede utilizadas e suas características, além da exibição do algoritmo de roteamento proposto. A Seção 4 se refere à avaliação do algoritmo. Por fim, a Seção 5 apresenta a conclusão do trabalho.

\section{Trabalhos Relacionados}

As SDM-EON têm motivado diversas pesquisas no desenvolvimento de algoritmos que utilizam os recursos da rede com mais eficiência. Controlar a fragmentação e o crosstalk médio é uma das maneiras mais utilizadas para melhorar a eficiência de utilização dos recursos. No entanto, poucos trabalham tem considerado estas características. Este trabalho considera a fragmentação e o crosstalk médio em conjunto para o problema de roteamento e alocação de modulação, espectro e núcleo para SDM-EON.

Em [Yousefi and Rahbar 2018] foram propostos três algoritmos que visam reduzir o problema da fragmentação e aumentar o número de conexões estabelecidas na rede. Os algoritmos FMMA-RSCA, MFPC e SBMC consideram diferentes prioridades na alocação: o primeiro considera a métrica de fragmentação Fragmentation Measure Metric (FMM) como principal condição; o segundo utiliza o núcleo mais fragmentado; e o terceiro toma os espaços no espectro como condição para escolha dos recursos. Os algoritmos utilizam a estratégia do multicaminho para redução da fragmentação porém não utilizam modulação adaptativa.

Em [Moghaddam et al. 2018], foram apresentadas uma Programação Linear Inteira (PLI) mista e uma heurística para lidar com o crosstalk e com problemas de agendamento. Considerou-se tráfego estático e as rotas foram previamente definidas (ou seja, de forma offline) pelo algoritmo de K-menores caminhos. A PLI mista garante que somente um caminho seja alocado para cada requisição, em seguida a modulação adaptativa é aplicada, e a condição de crosstalk é calculada para cada slot do espectro. A melhor modulação é selecionada a fim de mitigar a degradação do sinal pela distância e pela interferência do crosstalk. O objetivo é reduzir a quantidade de recursos diminuindo o número de slots alocados. A heurística objetiva equilibrar o uso de slots de frequência nos enlaces. Apesar da estratégia de redução de crosstalk adotada, não foram adotadas 
medidas de redução de fragmentação.

Em [Xiong et al. 2019], a abordagem do aprendizado de máquina foi utilizada para mitigar os problemas da fragmentação e do crosstalk nas SDM-EON. Para isso, foi utilizada uma rede neural para prever as demandas da rede, além de uma estratégia de visualização do espectro da rede de forma bidimensional horizontal para alocação do recurso. O trabalho propõe uma maneira de lidar principalmente com a fragmentação, no entanto, a abordagem não considera modulação adaptativa para melhor aproveitamento do espectro de frequência.

Em [Yin et al. 2019], foi introduzido um algoritmo para sobrevivência em SDMEON com ciência de crosstalk e adotando estratégia de multicaminho, considerando a proteção de multicaminho. Foi utilizada a abordagem de super-canais, utilizando economia de custo por meio da redução do número de slots de lasers necessários. Para encontrar o caminho foi utilizado o algoritmo de caminhos disjuntos de Bhandari de forma offline. Em seguida, para que a conexão seja estabelecida, a crosstalk é verificado e, caso o nível não seja aceitável, a conexão é negada. Os autores não consideraram o delay diferencial para roteamento multicaminho nem modulação adaptativa.

Em [Pederzolli et al. 2019], são apresentados dois algoritmos RSA que buscam minimizar a fragmentação. Por meio da utilização de diversas políticas de alocação são avaliadas as métricas que comprovam a efetividade dos algoritmos e definidas métricas de fragmentação que utilizam os slots não utilizados para calcular o estado do núcleo. Para minimizar a fragmentação são propostos dois algoritmos que são combinados posteriormente com políticas de alocação. Para os algoritmos, é utilizada somente a modulação QPSK para todas as transmissões.

Os autores em [Yousefi and Rahbar 2020] desenvolveram três algoritmos MINCROSS, MINFRAG e MODFRAGCROSS- para lidar com o crosstalk e manter o nível de segurança da camada física e reduzir a probabilidade de bloqueio. Os autores consideram a interferência causada pelo crosstalk entre núcleos. Há a tentativa de controlar a probabilidade de bloqueio por meio da prevenção de fragmentação e crosstalk. $\mathrm{O}$ algoritmo ExactFit é utilizado para encontrar espaços livres no espectro que podem alocar a exata quantidade de slots requisitados. O algoritmo MINCROSS prioriza a redução do crosstalk, o MINFRAG prioriza a redução de fragmentação, enquanto que o MODFRAGCROSS busca equilibrar crosstalk e fragmentação. Foram utilizadas as métricas de crosstalk por slot e fragmentação. Não foram utilizadas modulações adaptativas nem multicaminho, contribuindo para a não utilização do espectro da melhor forma.

O trabalho em [Yousefi et al. 2020] introduz duas métricas: Holding Time Metric (HTM) e Coefficient of the Variant Metric (CVM). Baseado nessas métricas, eles propuseram três algoritmos para resolver o problema de fragmentação e melhorar a probabilidade de bloqueio para o problema RSCA. Os algoritmos utilizam o desperdício de espectro para encontrar um retângulo para alocar cada conexão por meio do algoritmo K-menores caminhos, considerando a fragmentação para redução da taxa de fragmentação. Os autores não utilizam modulação adaptativa para os algoritmos propostos.

Em [Ujjwal et al. 2021], é proposto um esquema chamado Optimal Spectrum Defragmentation (DF), para dividir as conexões em uma estratégia para diminuir a fragmentação e aumentar o número de transmissões na rede. O caminho é encontrado por 
meio do algoritmo K-ShortestPath na política de alocação FirstFit (FF). Para conexões que não conseguem ser estabelecidas nesse modelo, é utilizado o esquema proposto. A multiplexação por divisão espacial não é considerada nesse trabalho, enquanto somente uma modulação é utilizada para as transmissões.

Tabela 1. Características dos trabalhos relacionados.

\begin{tabular}{ccccc}
\hline \multicolumn{1}{c}{ Trabalho } & SDM & $\begin{array}{c}\text { Modulação } \\
\text { Adaptativa }\end{array}$ & Crosstalk & Fragmentação \\
\hline [Yousefi and Rahbar 2018] & $\checkmark$ & & & $\checkmark$ \\
\hline [Moghaddam et al. 2018] & $\checkmark$ & $\checkmark$ & $\checkmark$ & \\
\hline$[$ [Xiong et al. 2019] & $\checkmark$ & & $\checkmark$ & $\checkmark$ \\
\hline [Yin et al. 2019] & $\checkmark$ & & $\checkmark$ & $\checkmark$ \\
{$[$ Pederzolli et al. 2019] } & & & $\checkmark$ & $\checkmark$ \\
{$[$ Yousefi and Rahbar 2020] } & $\checkmark$ & & $\checkmark$ & $\checkmark$ \\
\hline [Yousefi et al. 2020] & $\checkmark$ & & $\checkmark$ & $\checkmark$ \\
\hline [Ujjwal et al. 2021] & & & & \\
\hline REGARD & $\checkmark$ & $\checkmark$ & & \\
\hline
\end{tabular}

A Tabela 1 compara o algoritmo proposto neste artigo com vários outros descritos na literatura sobre o uso de multiplexação por divisão espacial, o uso de modulação adaptativa, a ciência de crosstalk e a ciência de fragmentação. Com base em nossa análise do estado da arte, concluímos que poucos estudos consideram o estudo de casos onde são combinadas todas as características de Redes Ópticas Elásticas mencionadas anteriormente. Até onde sabemos, este é o primeiro artigo a resolver o problema de roteamento, modulação, espectro e alocação de núcleo com ciência de crosstalk e fragmentação.

\section{Algoritmo}

Esta seção apresenta o Algoritmo de Roteamento e Alocação de Modulação, Núcleo e Espectro com Ciência de Fragmentação e Crosstalk (REGARD). O algoritmo tem como objetivo lidar com o problema RMSCA aumentando a eficiência de utilização de recursos e o número de conexões estabelecidas na rede. Durante o roteamento, formatos de modulações são aplicadas dinamicamente de acordo com a distância de transmissão (Tabela 2 ).

\subsection{Modelo de rede}

Foi adotado um modelo de rede óptica com utilização de multiplexadores ópticos reconfiguráveis flexíveis add/drop capaz de alternar entre comprimentos de onda e granularidade com transceivers MIMO (Multiple-Input Multiple Output). Também foi considerada a utilização de Transponders Multi-Flow (MFTs) para dividir em diversos sub transponders para facilitar a alocação.

A topologia de rede é composta por fibras bidirecionais com sete núcleos dispostos de forma hexagonal. Cada núcleo é composto por 320 slots de frequência com capacidade de transmissão de $12,5 \mathrm{GHz}$ cada. Foram utilizados os valores reais das distâncias entre pontos da topologia em quilômetros. Foram mantidas as restrições de continuidade e 
contiguidade, não sendo permitida a troca de slots e núcleos durante todos os enlaces do percurso. O cálculo do número de slots necessários para as transmissões é feito baseado na modulação aplicada, que por sua vez, é escolhida com base na distância ponto a ponto da transmissão. O número de slots para uma mesma demanda é menor caso a modulação aplicada seja maior. Além disso, o slot Filtro de Banda de Guarda (FGB) é sempre alocado para manter a separação entre o conjunto de slots de transmissões distintas.

Diversas modulações foram utilizadas para que ocorra a maior eficiência de utilização do espectro (Tabela 2). É importante que a modulação seja selecionada de forma adequada pois é essencial para a transmissão que o sinal seja decodificado corretamente. Para a seleção, a distância máxima que a modulação aplicada pode transmitir deve ser considerada pois esse limite permite que o sinal não sofra perdas e a decodificação seja feita de forma correta, por isso, a modulação mais eficiente deve ser escolhida para que o sinal mantenha qualidade.

Tabela 2. Características das modulações.

\begin{tabular}{cccc}
\hline $\begin{array}{c}\text { Nível de } \\
\text { Modulação }\end{array}$ & $\begin{array}{c}\text { \# Bits por } \\
\text { Símbolo }\end{array}$ & $\begin{array}{c}\text { Capacidade } \\
\text { do } \text { Slot } \mathbf{( G b / s )}\end{array}$ & $\begin{array}{c}\text { Distância } \\
\text { Máxima }(\mathbf{k m})\end{array}$ \\
\hline 64QAM & 6 & 75 & 125 \\
32QAM & 5 & 62.5 & 250 \\
16QAM & 4 & 50 & 500 \\
8QAM & 3 & 37.5 & 1000 \\
QPSK & 2 & 25 & 2000 \\
BPSK & 1 & 12.5 & 4000 \\
\hline
\end{tabular}

\subsection{Algoritmo REGARD}

O REGARD é um algoritmo RMSCA para SDM-EON, avaliado em diferentes cargas, cenários e topologias. O objetivo do REGARD é aumentar o número de conexões estabelecidas na rede e melhorar a eficiência na utilização dos recursos da rede, evitando desperdícios de slots de frequência após sucessivas conexões e desconexões. O algoritmo proposto não utiliza troca de núcleos durante a transmissão, dessa forma, a conversão óptico/elétrico/óptico não é necessária, mantendo as restrições de continuidade e contiguidade. Para aprimorar a alocação de recursos, foi considerado o estado dos núcleos em cada enlace da rede. A avaliação foi feita por meio da computação dos níveis de fragmentação em cada núcleo e crosstalk médio entre núcleos. O impacto das novas requisições foi estimado para que a fragmentação e o crosstalk se mantivessem em níveis adequados, além de estabelecer o maior número de conexões possíveis na rede.

O processo se inicia por meio do algoritmo responsável pelo cálculo de rotas offine. Para cada par de nós da rede são calculadas as rotas por meio do algoritmo $K$ ShortestPath, com K igual a 15 caminhos não necessariamente disjuntos, de modo a existir maior possibilidade de uma das rotas ser apropriada à requisição, em seguida, são armazenados em uma matriz com índices correspondente ao par de nós. A matriz de rotas resultante é utilizada na execução do algoritmo REGARD para roteamento e alocação de recursos.

O Algoritmo 1 apresenta as tomadas de decisão para o roteamento e alocação de recursos. O resultado do algoritmo de rotas offline é utilizado como entrada neste 


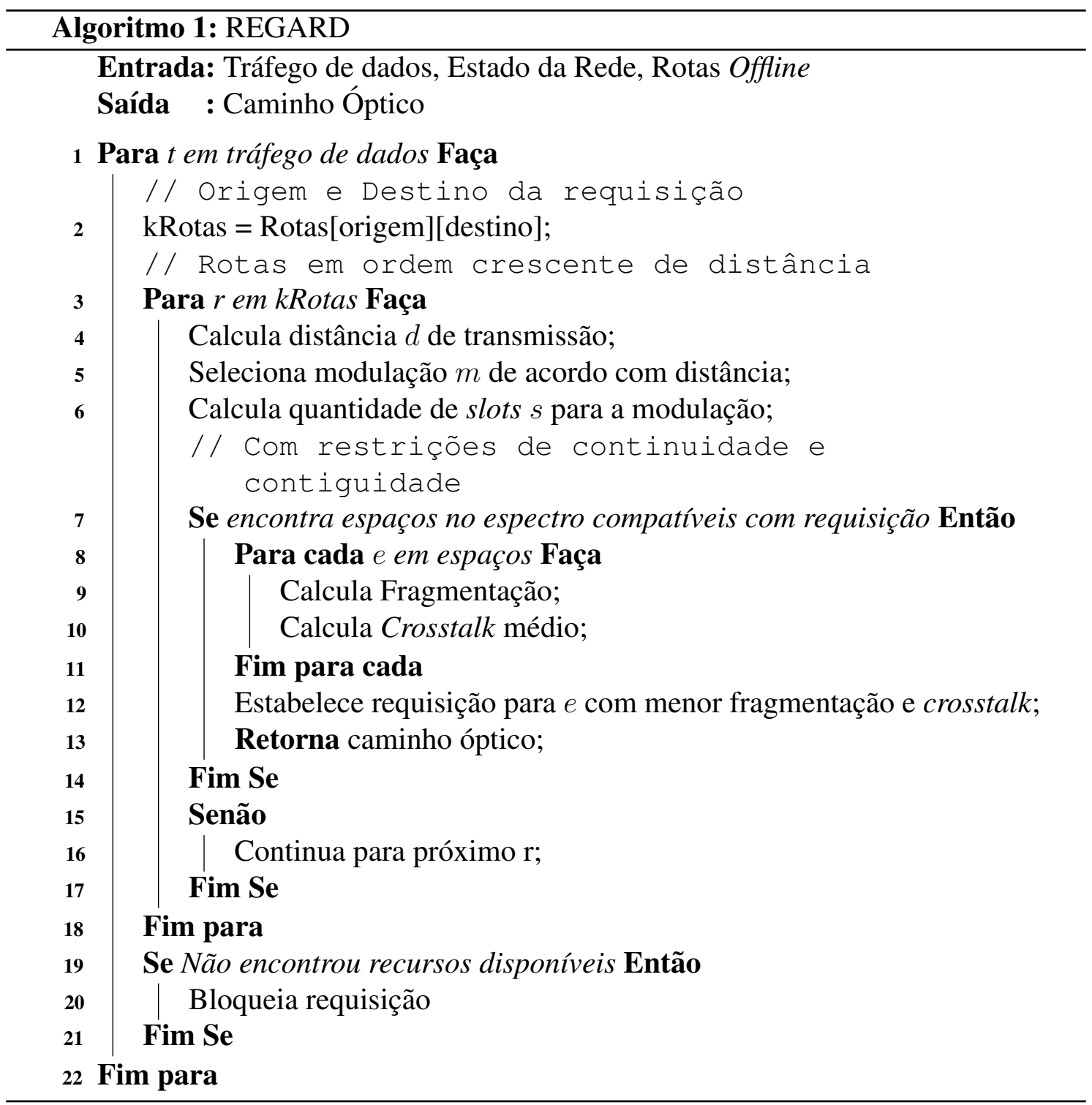

momento, assim como o tráfego de dados, com as requisições ordenadas em tempo de chegada à rede. Para cada uma das requisições, são verificados os nós de origem e destino da transmissão, e as rotas são obtidas de acordo com a matriz predefinida de rotas (Linha 2). Considerando estas rotas em ordem crescente de distância (Linhas 3), faz-se a busca por recursos ópticos disponíveis até que uma rota $r$ tenha recursos suficientes para provisionar a requisição. Nas Linhas 4-6, calcula-se a distância do percurso a fim de se definir a modulação aplicada e calcular o número de slots necessários, somados ao FGB. Em seguida, na Linha 7 está descrito o processo de verificação de disponibilidade do espectro para cada um dos enlaces do percurso. Os espaços que foram encontrados e que mantém as restrições de continuidade e contiguidade são utilizados para calcular a fragmentação (Linha 9) e o crosstalk médio (Linha 10). O crosstalk é calculado por meio do número de transmissões que sofrem interferência em núcleos adjacentes, enquanto a fragmentação é calculada pela métrica FMM (Fragmentation Measure Metric) proposta pela Equação (3) em [Yousefi et al. 2019]. Os recursos são então alocados para o conjunto de slots que oferecem menor impacto à rede, tanto com menor interferência, quanto com 
menor fragmentação (Linha 12). Para os casos onde não existem recursos para acomodar a requisição, ela é então bloqueada (Linhas 19-20). Esse processo é repetido para todo o tráfego da rede (Linha 1).

\section{Avaliação}

Nesta seção, é feita a avaliação de desempenho do algoritmo REGARD, além de uma análise comparativa com outros algoritmos presentes na literatura por meio de simulações de diversos parâmetros e cenários envolvendo SDM-EON.

\subsection{Simulação e Métricas}

Para análise de performance do algoritmo RMSCA foram feitas simulações em SDMEON utilizando o simulador de eventos discretos Flexgridsim [Moura and Drummond]. A geração de requisições seguiram o processo de Poisson e foram distribuídos uniformemente entre os nós da rede. Para cada cenário foram replicadas 10 simulações com carga de rede variando entre 50 e 1000 erlangs, com 100.000 requisições para cada simulação feita. Intervalos de confiança foram gerados por meio do método de replicação, sendo adotado o nível de confiança de $95 \%$. Para os diferentes algoritmos, foi utilizado o mesmo conjunto de sementes. Foram utilizadas 7 diferentes tipos de requisições com taxas de transmissão de 25/50/125/200/500/750/1000 Gbps igualmente proporcionais. A fibra utilizada no trabalho é composta por 7 núcleos arranjados de forma hexagonal, com divisão de 320 slots de frequência para cada núcleo. As topologias utilizadas nas simulações foram a topologia USA (Fig 1(a)], com 24 nós e 43 enlaces, e a topologia NSF ( Fig 1(b)], com 14 nós e 19 enlaces, duas das principais topologias existentes. O objetivo de utilizar tais topologias é retratar uma situação com topologias com distâncias reais entre os nós, representado pelo . O número em cada enlace das topologias representa a distância entre nós em quilômetros.

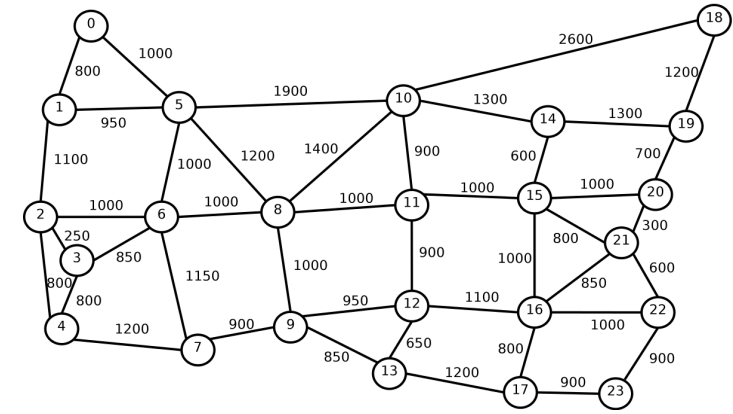

(a) USA

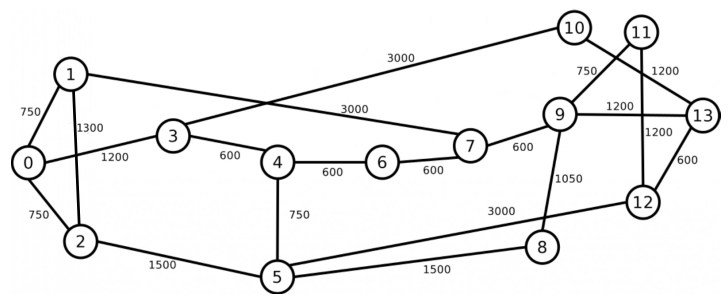

(b) NSF

Figura 1. Topologias.

As métricas utilizadas para avaliação de desempenho dos algoritmos foram Taxa de Bloqueio de Banda (BBR), Crosstalk por Slot (CpS), Eficiência Energética (EE), Taxa de Fragmentação (FR), Porcentagem de Formatos de Modulação (MFP) e número médio de saltos.

O $B B R$ é calculado pela soma de toda banda bloqueada $(\alpha)$ durante o período de simulação, dividido pela soma de toda banda das requisições $(\beta)$ pelo mesmo período de tempo (Equação (1)). 


$$
B B R=\frac{\sum \alpha}{\sum \beta}
$$

O $C p S$ (Equação (2)) é o resultado da divisão do número de slots alocados sobre o mesmo espectro de frequência $(\gamma)$ no núcleo utilizado e adjacentes, pelo total de slots utilizados no enlance $(\varphi)$, calculado em divisões periódicas $(T)$ de mesmo intervalo.

$$
C p S=\frac{\sum \frac{\sum \gamma}{\sum \varphi}}{T}
$$

A $E E$ é calculada pela divisão entre a soma da largura de banda (em Mbps) de todas as requisições aceitas na rede $(\gamma)$ e a soma do consumo energético (em Joules) dos transponders, comutadores e amplificadores ópticos presentes no caminho das requisições aceitas, como descrito na Seção 3. A EE é definida pela Equação (3).

$$
E E=\frac{\sum \gamma}{\sum \epsilon}
$$

O FR (Equação (4)) é calculado pela divisão do número de blocos de slots $(\mu)$ disponíveis pelo número de slots disponíveis no enlace $(\delta)$.

$$
F R=\frac{\operatorname{Max}\left(\sum \mu\right)}{\sum \delta}
$$

O MFP é calculado pela quantidade de vezes que cada modulação foi utilizada pelas transmissões $\left(T_{m}\right)$ dividido pelo número total de transmissões (Total). O MFP é definido pela Equação (5).

$$
M F P=\frac{T_{m}}{\text { Total }}
$$

\subsection{Resultados}

Nesta seção são apresentados os gráficos resultantes para o algoritmo proposto REGARD. Para avaliar o desempenho do algoritmo proposto, são realizadas comparações com os algoritmos MINCROSS [Yousefi and Rahbar 2020], MINFRAG [Yousefi and Rahbar 2020] e MINIMIZE [Pederzolli et al. 2019].

As Figuras 2(a) e 2(b) apresentam os resultados de BBR em função da carga na rede para as topologias USA e NSF, respectivamente. Para topologia USA, os algoritmos MINCROSS e MINFRAG bloqueiam a partir de 50 erlangs, o algoritmo MINIMIZE bloqueia a partir de 100 erlangs, enquanto o algoritmo REGARD bloqueia requisições a partir de 200 erlangs. Para a topologia NSF, MINCROSS e MINFRAG bloqueiam requisições a partir da carga de 50 erlangs e os algoritmos MINIMIZE e REGARD bloqueiam requisições a partir de 100 erlangs. Para cargas acima de 500 erlangs, os resultados tornam-se bastante próximos, com taxas de bloqueio mais altas devido a carga da rede. A estratégia adotada pelo algoritmo REGARD para a busca de caminhos disponíveis 


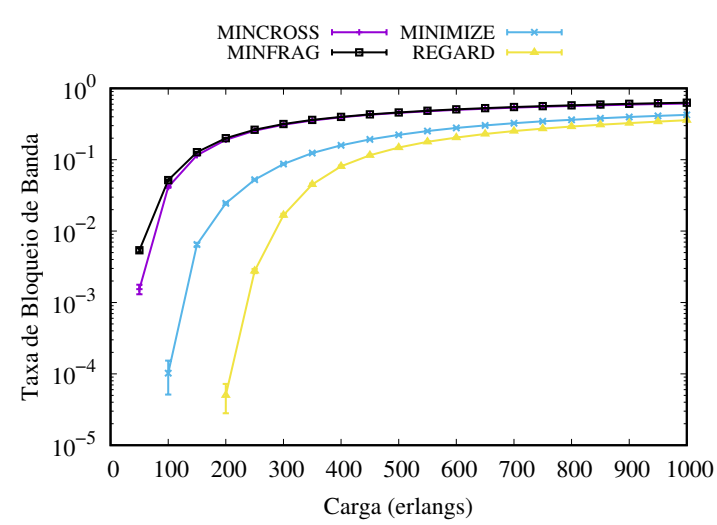

(a) USA

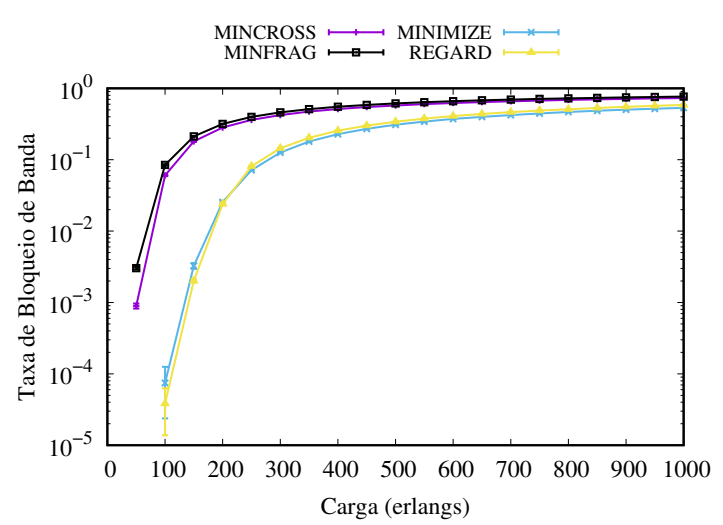

(b) NSF

Figura 2. Probabilidade de Bloqueio de Banda (BBR).

para acomodar as requisições mostrou maior eficiência dentre os comparados, comprovado pela BBR mais baixa por todas as cargas simuladas. As rotas que são calculadas entre os nós da rede proporcionam maior probabilidade de dispor um caminho com recursos suficientes para a requisição devido a quantidade de rotas entre os pares da rede. A topologia USA apresenta maior conectividade que a topologia NSF, proporcionando um número maior de rotas alternativas e, consequentemente, BBR mais baixo sob as mesmas cargas.

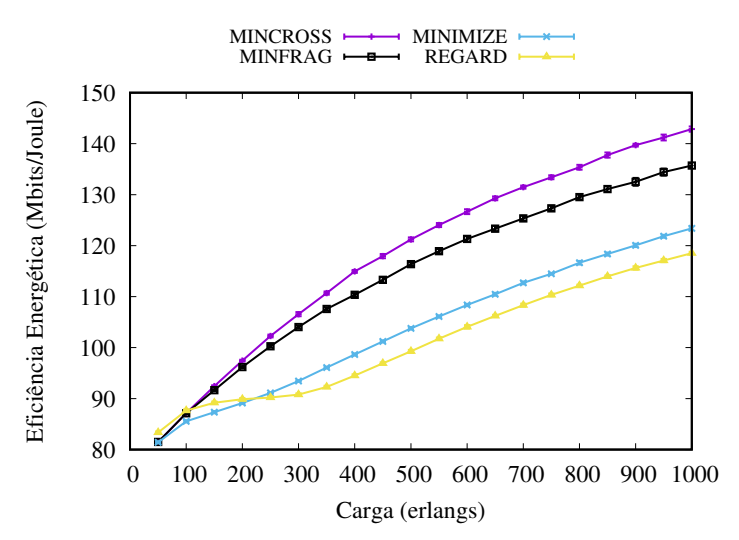

(a) USA

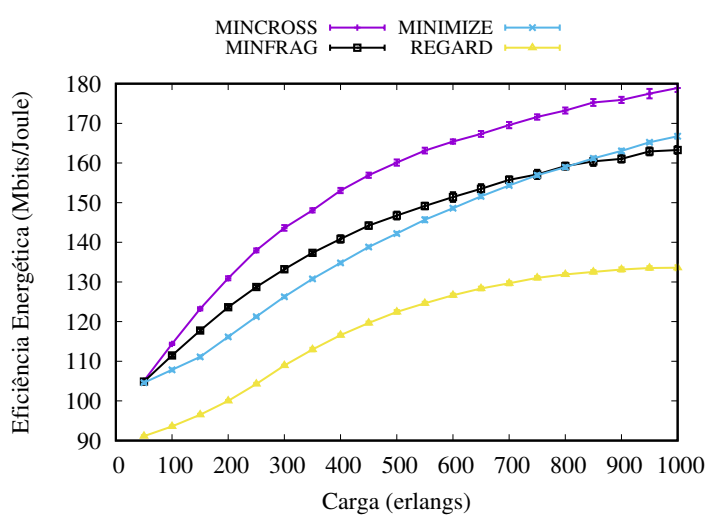

(b) NSF

Figura 3. Eficiência Energética (EE).

As Figuras 3(a) e 3(b) exibem a eficiência energética em função da carga na rede para algoritmos comparados. Para a topologia USA, MINCROSS apresenta maior eficiência energética, seguido de MINFRAG com eficiência energética próxima, em seguida o algoritmo MINIMIZE e, com menor eficiência energética, o algoritmo REGARD. Para a topologia NSF o comportamento é semelhante, com MINCROSS apresentando maior eficiência energética, em segundo o algoritmo MINFRAG e em terceiro o algoritmo MINIMIZE, todos próximos entre si, no entanto, com a menor eficiência energética aparece o algoritmo REGARD. O algoritmo REGARD apresenta baixa eficiência energética devido o custo de energia para as transmissões ser alto, consequência do alto número médio de saltos para as transmissões (Figura 4). 


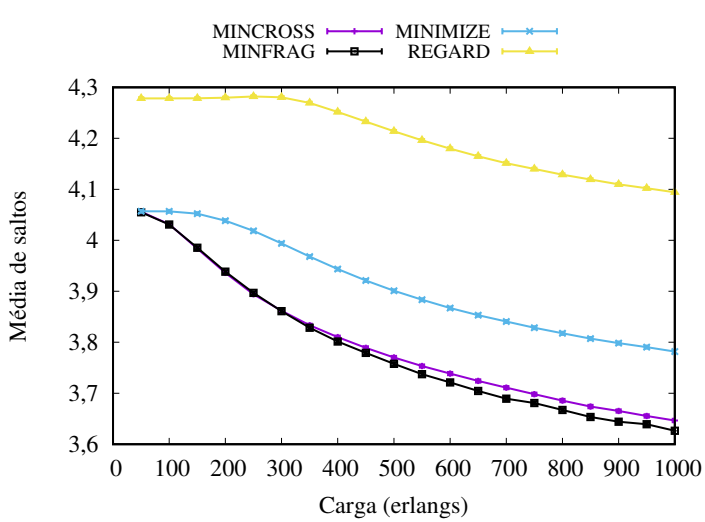

(a) USA

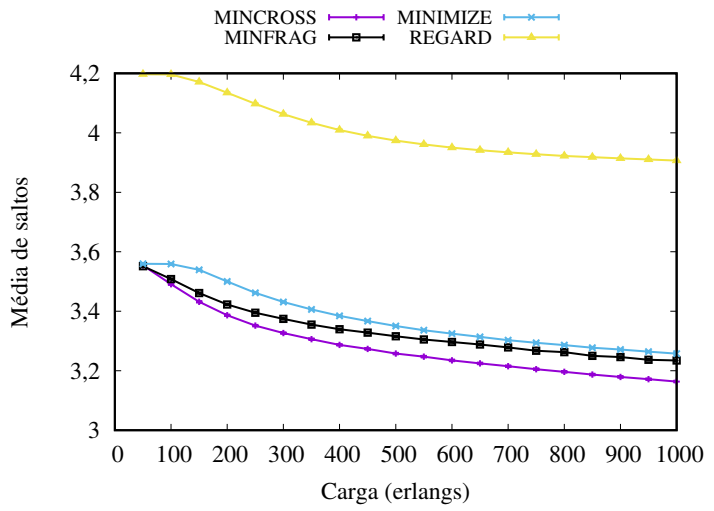

(b) NSF

Figura 4. Número Médio de Saltos.

As Figuras 4(a) e 4(b) apresentam o número médio de saltos em função da carga para as transmissões estabelecidas na rede. Os algoritmos MINCROSS e MINFRAG apresentam média de saltos bastante próximas para todas as cargas simuladas, enquanto o MINIMIZE apresenta média de saltos superior a MINCROSS e inferior a REGARD. O algoritmo REGARD apresenta a maior média de saltos para todas as cargas devido o mecanismo utilizado para encontrar o caminho para a requisição. Diferentemente dos algoritmos usados na comparação, que usam o menor caminho, o mecanismo de busca de caminhos do algoritmo REGARD busca vários caminhos possíveis entre dois nós, desde caminhos mais curtos e com menos saltos a caminhos mais longos e com mais saltos. Isso acontece para que se possa garantir menores níveis de fragmentação e de crosstalk. Os caminhos mais curtos são os primeiros a serem verificados, no entanto, para os casos onde eles não estão disponíveis, caminhos alternativos precisam ser alocados, selecionando caminhos com maior número de saltos e, consequentemente, maior gasto energético.

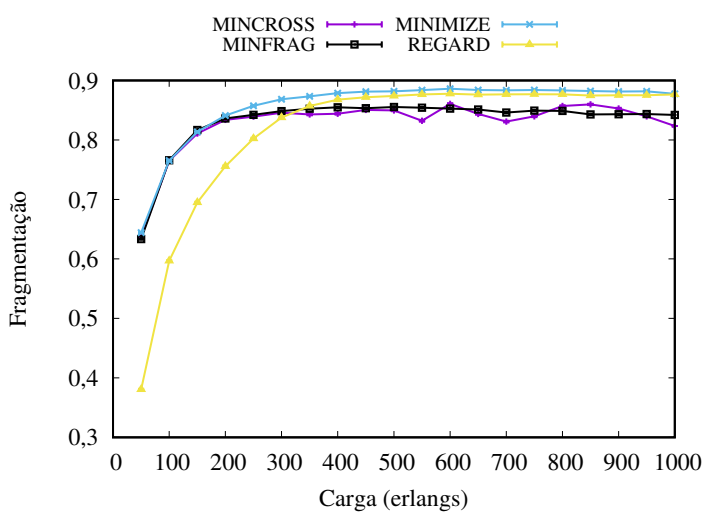

(a) USA

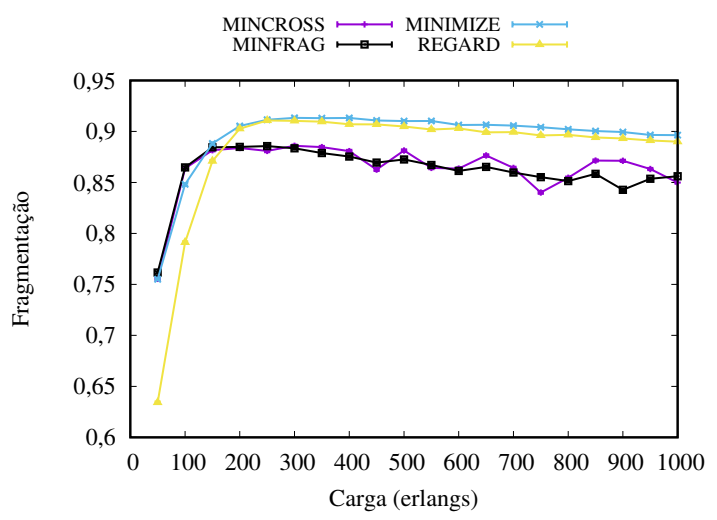

(b) NSF

Figura 5. Relação de Fragmentação (FR).

As figuras 5(a) e 5(b) apresentam a relação de fragmentação em função da carga na rede para os algoritmos comparados. Para topologia USA, os algoritmos MINCROSS, MINFRAG e MINIMIZE apresentam níveis de fragmentação próximos até a carga de 
200 erlangs, sendo superiores ao nível de fragmentação de REGARD. Após a carga de 200 erlangs, o nível de fragmentação de REGARD supera MINCROSS e MINFRAG. De forma semelhante para a NSF, o nível de fragmentação de REGARD permanece inferior até a carga de 150 erlangs, superando a fragmentação de MINFRAG e MINCROSS após tal carga. O aumento da fragmentação de REGARD sobre os outros algoritmos se deve ao fato de a média de saltos ser alta, consequentemente, para as cargas mais altas, grande número de slots são utilizados para uma transmissão.

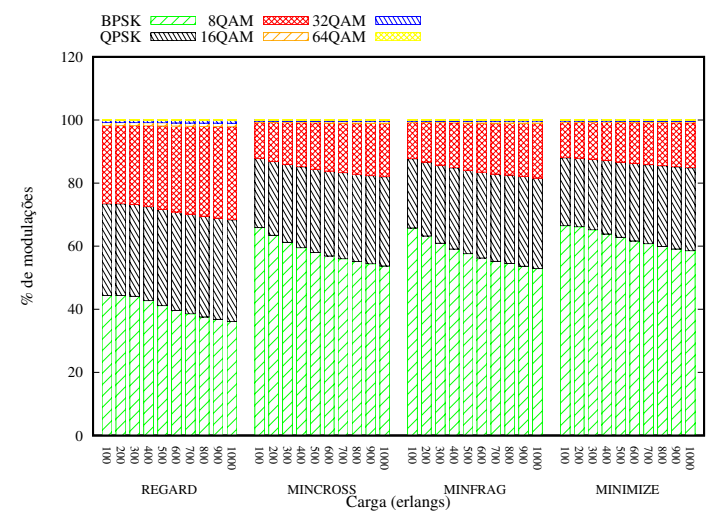

(a) USA

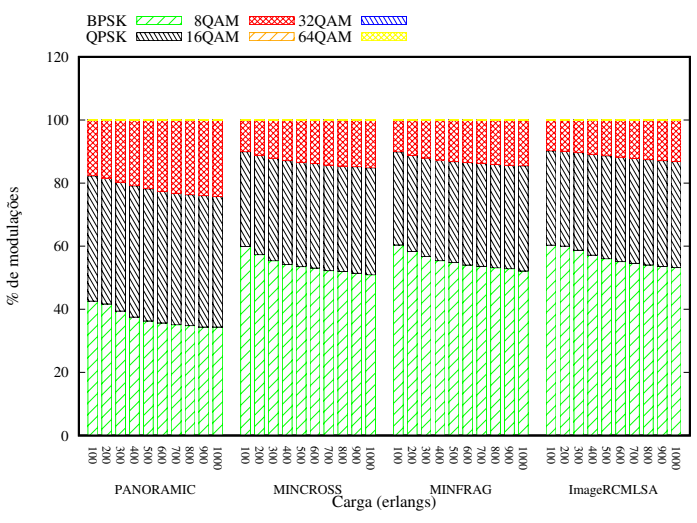

(b) NSF

Figura 6. Relação de Modulações Aplicadas (MFP).

As Figuras 6(a) e 6(b) mostram a porcentagem de modulações utilizadas nas simulações de cada algoritmo. A modulação aplicada considera a distância total de transmissão, que sofre influência tanto da topologia quanto das requisições. Para a topologia USA, a modulação mais utilizada é a BPSK, por suportar maior distância de transmissão. As modulações QPSK e 8QAM apresentam porcentagem expressiva de uso por suportar médias distâncias de transmissão, enquanto as modulações 16QAM e 32QAM são pouco utilizadas devido característica da topologia, que apresentam somente dois enlaces com distância adequada para aplicar tais modulações, enquanto a modulação 64QAM não é utilizada. Para a topologia NSF, a modulação mais utilizada é a BPSK, com QPSK em segundo em taxa de utilização e, por último, a modulação 8QAM. As modulações 16QAM, 32QAM e 64QAM não são utilizadas devido característica da topologia, que não possui enlaces com distância suportadas por estas modulações. O algoritmo REGARD apresenta modulações com taxa de utilização mais distribuída devido a característica de buscar maior quantidade de rotas e verificar qual é mais adequada, sempre priorizando rotas de menor distância e capazes de aplicar maior nível de modulação quanto possível, consistindo no melhor aproveitamento dos slots quando comparado aos algoritmos MINCROSS, MINFRAG e MINIMIZE.

\section{Conclusão}

Este artigo apresenta REGARD, um algoritmo RMSCA que utiliza modulação adaptativa e ciência de crosstalk e fragmentação para melhor alocação de recursos. O algoritmo REGARD reduz o bloqueio de banda e melhora a eficiência de alocação. O algoritmo faz uma busca offline de rotas de um nó a outro para reduzir o tempo de cálculo de caminho durante a chegada da requisição à rede. O algoritmo REGARD favorece maior 
quantidade de dados transmitidos na rede pela baixa taxa de bloqueio para cargas mais baixas, enquanto mantém os níveis de crosstalk e fragmentação adequados, combinados à utilização de modulações mais eficientes para as transmissões.

\section{Agradecimentos}

Este trabalho foi financiado pela bolsa processo no 2020/05054-5 da Fundação de Amparo à Pesquisa do Estado de São Paulo (FAPESP).

\section{Referências}

Bonani, L., Queiroz, J., Abbade, M., and Callegati, F. (2019). Load balancing in fixedrouting optical networks with weighted ordering heuristics. IEEE/OSA Journal of Optical Communications and Networking, 11(3):26-38.

Cisco, U. (2018). Cisco Visual Networking Index (VNI). Complete Forecast Update, 2017-2022. https://www.cisco.com/c/ $\mathrm{dam} / \mathrm{m} /$ en_us/network-intelligence/service-provider/ digital-transformation/knowledge-network-webinars/pdfs / 1213-business-services-ckn.pdf. Último acesso: 14/04/2021.

Iyer, S. (2020). On routing, modulation format, space and spectrum allocation with protection in space division multiplexing-based elastic optical networks. Journal of Information and Telecommunication, 4(1):105-117.

Mendinueta, J. M. D., Shinada, S., Hirota, Y., Furukawa, H., and Wada, N. (2020). Highcapacity super-channel-enabled multi-core fiber optical switching system for converged inter/intra data center and edge optical networks. IEEE Journal of Selected Topics in Quantum Electronics, 26(4):1-13.

Moghaddam, E. E., Beyranvand, H., and Salehi, J. A. (2018). Crosstalk-aware routing, modulation level, core and spectrum assignment, and scheduling in SDM-based elastic optical networks. In International Symposium on Telecommunications (IST), pages $160-165$.

Moura, P. M. and Drummond, A. C. FlexGridSim: Flexible Grid Optical Network Simulator. http://www.Irc.ic.unicamp.br/FlexGridSim/, Último acesso: $14 / 04 / 2021$.

Oliveira, H. M. and da Fonseca, N. L. (2019). Protection, routing, spectrum and core allocation in EONs-SDM for efficient spectrum utilization. In IEEE International Conference on Communications (ICC), pages 1-6. IEEE.

Paira, S., Halder, J., Chatterjee, M., and Bhattacharya, U. (2020). On energy efficient survivable multipath based approaches in space division multiplexing elastic optical network: Crosstalk-aware and fragmentation-aware. IEEE Access, 8:47344-47356.

Pederzolli, F., Siracusa, D., Zanardi, A., Galimberti, G., Fauci, D. L., and Martinelli, G. (2019). Path-based fragmentation metric and RSA algorithms for elastic optical networks. IEEE/OSA Journal of Optical Communications and Networking, 11(3):1525 .

Secondini, M. (2020). Information capacity of optical channels. In Optical Fiber Telecommunications VII, pages 867-920. Elsevier. 
Trindade, S. and da Fonseca, N. (2020). Resource allocation in space-division multiplexing-based elastic optical networks. In Anais Estendidos do XXXVIII Simpósio Brasileiro de Redes de Computadores e Sistemas Distribuídos, pages 113-120, Porto Alegre, RS, Brasil. SBC.

Ujjwal, Thangaraj, J., and Rajnish kumar (2021). Multi-path provisioning in elastic optical network with dynamic on-request optimal defragmentation strategy. Optical Switching and Networking, 41:100607.

Winzer, P. J. (2020). Transmission system capacity scaling through space-division multiplexing: a techno-economic perspective. In Optical Fiber Telecommunications VII, pages 337-369. Elsevier.

Xiong, Y., Yang, Y., Ye, Y., and Rouskas, G. N. (2019). A machine learning approach to mitigating fragmentation and crosstalk in space division multiplexing elastic optical networks. Optical Fiber Technology, 50:99-107.

Xiong, Y., Ye, Y., Zhang, H., He, J., Wang, B., and Yang, K. (2020). Deep learning and hierarchical graph-assisted crosstalk-aware fragmentation avoidance strategy in space division multiplexing elastic optical networks. Opt. Express, 28(3):2758-2777.

Yin, S., Zhang, Z., Chen, Y., Ma, R., and Huang, S. (2019). A survivable xt-aware multipath strategy for SDM-EONs. In Asia Communications and Photonics Conference $(A C P)$, pages $1-3$.

Yousefi, F., Ghaffarpour Rahbar, A., and Ghadesi, A. (2020). Fragmentation and time aware algorithms in spectrum and spatial assignment for space division multiplexed elastic optical networks (SDM-EON). Computer Networks, 174:107232.

Yousefi, F., Ghaffarpour Rahbar, A., and Yaghubi-Namaad, M. (2019). Fragmentationaware algorithms for multipath routing and spectrum assignment in elastic optical networks. Optical Fiber Technology, 53:102019.

Yousefi, F. and Rahbar, A. G. (2018). Novel fragmentation-aware algorithms for multipath routing and spectrum assignment in elastic optical networks-space division multiplexing (EON-SDM). Optical Fiber Technology, 46:287-296.

Yousefi, F. and Rahbar, A. G. (2020). Novel crosstalk, fragmentation-aware algorithms in space division multiplexed- elastic optical networks (SDM-EON) with considering physical layer security. Optical Switching and Networking, 37:100566.

Zhu, R., Samuel, A., Wang, P., Li, S., Oun, B. K., Li, L., Lv, P., Xu, M., and Yu, S. (2021). Protected resource allocation in space division multiplexing-elastic optical networks with fluctuating traffic. Journal of Network and Computer Applications, 174:102887. 